\title{
The Challenge of Using SNPs in the Understanding and Treatment of Disease
}

Richard S. Schifreen ${ }^{1}$, Douglas R. Storts ${ }^{1}$, and Arlene M. Buller ${ }^{2}$

${ }^{1}$ Promega Corporation, Madison, WI and ${ }^{2}$ Quest Diagnostics, San Juan Capistrano, CA, USA

BioTechniques 32: S14-S21 (June 2002)

\section{DISCOVERING USEFUL SNPs}

\section{Overview of Current Efforts to Discover Useful SNPs}

A substantial private and public effort has been undertaken to characterize the SNPs responsible for genetic diversity. The ultimate goal is to identify, map, and determine the allele frequency of a useful set of SNPs that are evenly distributed along the genome. These SNPs represent potentially valuable tools for mapping disease genes, predicting drug metabolism, increasing our understanding of population genetics, identifying polymorphisms that are useful for diagnosing genetic disorders - or predisposition to disease, and improving our understanding of other complex biological processes. In response to a need to develop faster and lower cost methods for evaluating SNPs, a diverse group of academic and industrial researchers have developed new approaches, or modifications to existing approaches, for SNP identification and analysis.

The SNP Consortium, consisting of the Wellcome Trust and approximately one dozen participating companies, was established in 1999 with the goal of identifying 300000 mapped SNPs. The premise for the consortium was to provide "publicly available and freely accessible" information to enable advancements in the understanding of human disease. As of early 2002, the consortium has released 1.25 million mapped SNPs (8).

The National Center for Biotechnology Information (NCBI) maintains a database of SNPs (dbSNP) submitted by $\mathrm{NIH}$-funded researchers, as well as contributions by other individuals and organizations (9). As of February 2002, this database included 4.1 million SNPs.

In addition to these two nonprofit organizations, several commercial and public entities have undertaken projects to identify informative SNPs, create high-density SNP maps, and determine the allele frequency (the frequency of occurrence) in different populations. These organizations rely on a variety of methods to detect SNPs (mass spectroscopy, bioluminescent detection, or fluorescent detection on arrays, particles, capillary electrophoresis devices, etc.), but most are based on allele-specific hybridization, or polymerase-dependent extension of a primer located adjacent to the presumptive polymorphism (18).

SNP discovery, the process of identifying new SNPs, is typically accomplished by DNA sequence analysis of pooled DNA isolated from multiple individuals. Apparent variations in the sequences of the pooled DNA, as represented by the appearance of two nucleotides at the same position, are subjected to additional confirmation. The process used for SNP validation typically involves PCR amplification of the region surrounding the presumptive polymorphism using DNA isolated from individuals represented in the pooled samples. These amplification products are then subjected to DNA sequence analysis to confirm (validate) the presence of the polymorphism.

\section{SNP Association Versus Functional Mutations}

SNPs may be located within coding regions (cSNPs) or map outside of coding regions. The cSNPs may result in mutations affecting protein function or result in neutral mutations that do not affect the protein function. Others (associated SNPs) map outside of the coding regions, but may serve as useful markers because of their proximity to disease loci or loci associated with drug metabolism, or because of effects on the regulation of transcription. Associated SNPs, while not the causative agent of the phenotype, can be used for detailed mapping of the chromosome to pinpoint the mutation(s) that are actually responsible for the disease phenotype.

\section{Mapping Useful SNPs}

After identifying and validating the SNP, the polymorphism is mapped to a specific region of the genome. Mapping the SNP to a specific region of the genome is accomplished by radiation hybrid mapping (13), in those instances where the genome sequence is unavailable. The completed genome sequence represents the most accurate and precise map. Mapping the SNP by comparing the DNA sequence of the region surrounding the SNP to the "reference" human genome sequence is referred to as an in silico approach.

The next step in the process is to measure the percentage of a population that contains the polymorphism (i.e., the allele frequency). Frequency data is routinely obtained by determining the percentage of individuals within a population that contains the polymorphism. Confirmed polymorphisms that occur at a high frequency (e.g., $>5 \%-10 \%$ ) represent potentially useful SNPs. Those SNPs that occur at lower frequencies, although potentially useful, are frequently discounted because of the problems inherent in obtaining statistically significant data from reasonably sized sample populations. There have been recent reports of new approaches for determining allele frequency using pooled 
DNA samples, reducing the requirement for genotyping from multiple individuals to estimate frequency $(1,11)$. Researchers at Promega have also demonstrated approaches to estimate allele frequencies from pooled samples (unpublished observation).

\section{Haplotypes}

It is estimated there may be as many as 10 million SNPs in the human genome, assuming a SNP frequency of approximately 1:1000 nucleotides. This observation quickly raised the question of how many SNPs are required to perform association studies. Genome-wide scans of all potential SNPs from a statistically meaningful population is impractical given the existing throughput limitations and assay costs. Assuming an average SNP assay cost of $\$ 0.50$ (probably represents an underestimate of all the current costs associated with SNP genotyping), a single study involving 100000 SNPs in 1000 individuals would cost \$50 million. Consequently, there is considerable effort focused on identification of a compromise approach: sufficient marker density to identify associations while minimizing the cost and effort.

The genome recombines in large blocks, or stretches, of DNA. This observation led researchers to conclude that closely linked SNPs are inherited in these large blocks (15), a process referred to as linkage disequilibrium (LD). Simply stated, ten SNPs that reside within the same LD block will be co-inherited. Consequently, any one of the 10 SNPs serves as a marker for the entire group of $10 \mathrm{SNPs}$ (i.e., they can be considered a single haplotype). Grouping SNPs based on LD will reduce the number of SNPs required to map disease gene loci, or other genes of interest, as evidenced by several recent publications $(3,10,16)$. Although haplotypes will likely play an increasingly important role in mapping studies, it is becoming clear that LD is not consistent across the genome or within specific regions of the genome across various populations $(14,15)$. Consequently, additional studies are required to interpret the complex patterns of $\mathrm{LD}$.

\section{SNP Genotyping Technology}

The discovery and study of SNPs have been facilitated by the development and commercial introduction of powerful new automated techniques. Traditionally, SNPs could be identified through sequencing or through gel-based methodologies such as restriction fragment length polymorphisms (RFLPs) and allelicspecific PCR. These technologies, while effective, are relatively slow and expensive. They also require subjective interpretation of gels that can be an additional source of error. Two general families of technologies have emerged to facilitate SNP genotyping. One family is focused on the high-throughput, multiplexed assay needs for performing the thousands of genotypes required for association and LD studies. These technologies use robotics and parallel detection technologies to increase throughput and reduce cost. They include the automated robotic versions of SNP-IT TM technology products being offered by Orchid BioSciences (Princeton, NJ, USA) based on their primer extension technology and the Sequenom mass spectrometry based MassARRAYTM system (San Diego, CA, USA). Other companies offer SNP genotyping as a service model, including the Qiagen Genomics mass spectrometry based Masscode ${ }^{\mathrm{TM}}$ system (Qiagen, Valencia, CA, USA) and the Orchid MegaSNPatron facility services. Third
Wave also participates in this market through design and synthesis services to support its Invader ${ }^{\circledR}$ technology (Third Wave Technologies, Madison, WI, USA). In addition, recent improvements in high-throughput capillary sequencing have made it a viable option.

There is also a need for lower throughput assays focused on SNP scoring applications. These technologies are best applied to repetitive analysis of a small group of SNPs once they have been identified. They are well-suited for scoring the genotypes of a small number of SNPs to support clinical research, validation studies, and molecular diagnostics. These technologies include the Promega READIT ${ }^{\circledR}$ SNP Genotyping System, the Pyrosequencing PSQ ${ }^{\mathrm{TM}} 96$ system (Uppsala, Sweden), Third Wave Technologies' Invader Assays, and the version of the Orchid technology being offered by Applied Biosystems as the PRISM ${ }^{\circledR}$ SNaPshot ${ }^{\mathrm{TM}}$ Multiplex System (Foster City, CA, USA). In addition, real-time PCR systems such as the Applied Biosystems PRISM Sequence Detection Systems for TaqMan ${ }^{\circledR}$ Technology, the Intergen Amplifluor ${ }^{\mathrm{TM}}$ system (Atlanta, GA, USA), and molecular beacons have been successfully applied to SNP scoring on a number of different instrument platforms.

\section{Using SNPs as a Diagnostic Tool}

Now that more than three million or more SNPs have been cataloged, the focus is shifting toward practical applications. This trend is facilitated by the commercial introduction of convenient and reliable assay technologies such as the Promega READIT SNP Genotyping System. Current estimates are that only 2000 (less than 1\%) of identified SNPs represent a change in amino acid sequence or a regulatory site that will affect protein expression. Not all alterations in protein expression will result in phenotypic changes or in clinically significant disease. In many cases, clinically useful SNPs have been identified based on biochemical studies. Consider the case of sickle cell anemia, where protein biochemistry was used to identify the functional glutamic acid to valine mutation in the hemoglobin beta chain long before the availability of SNP databases or genotyping technologies. In fact, the top five genetic tests performed by molecular diagnostic laboratories - cystic fibrosis, factor V Leiden, hemochromatosis, prothrombin (factor II), and fragile Xare all related to diseases that had been characterized prior to development of genetic databases. It is uncertain how many new diseases will be found related to discovery of SNPs. Coupled with the challenge of establishing the clinical utility of a SNP assay is the ability to perform the assay in a cost-effective manner. Analysis of several representative tests performed at a major reference laboratory (Table 1) indicates that assays brought online incorporate clinical utility as well as significant volume and earnings potential. Perhaps the greatest potential of SNPs - and the greatest challenge-will be developing their cost-effective use as markers for susceptibility to particular disorders or as predictors of response to environmental factors or pharmaceuticals.

\section{Functional SNPs in Hereditary Thrombophilia}

The most obvious candidates for diagnostically useful SNPs will be those that encode changes in amino acid sequence or a regulatory site. In some cases, such as sickle cell anemia, the 
mutation itself will be sufficient to diagnose a disease phenotype. With most SNP-related diseases, determining clinical utility is much more complex. Multiple pathways that interact among themselves and with environmental stimuli often determine metabolic functions. The diagnosis of hereditary thrombophilia is one of the most common applications for SNP testing in today's clinical diagnostic laboratories. Hereditary thrombophilia is defined as a tendency toward venous thrombotic disease in the absence of known acquired risk factors such as surgery, malignancy, or inactivity. It is known to be associated with point mutations in the genes coding for coagulation factors $\mathrm{V}$ and II (prothrombin). The factor $\mathrm{V}$ mutation (R506Q), referred to as factor V Leiden, results from a guanidine to adenine substitution at nucleotide 1691 in exon 10 of the FV gene (19). This mutation results in substitution of an arginine by glutamine at the site of proteolytic degradation by activated protein $\mathrm{C}$, a regulatory control on excess factor $\mathrm{V}$ activity. This mutation is found in 3\% to 5\% of apparently normal people and up to $50 \%$ of patients with a family history of thrombotic disease. It is found almost exclusively in the Caucasian population.

A thrombophilic single base mutation has also been identified in the $3^{\prime}$ untranslated region of the factor II or prothrombin gene $(\mathrm{G} 2021 \mathrm{~A} ; 4)$. The mechanism for how this mutation results in an affected phenotype is not yet known. The prevalence of this mutation in the general population is estimated at $2 \%$, and it increases the risk of deep venous thrombosis by $2.7 \%$ to $3.8 \%$. In addition, a SNP mutation in the methyenetetrahydrofolate reductase gene (MTHFR) has also been associated with hereditary thrombophilia.

Diagnostic assays for both the factor $\mathrm{V}$ Leiden mutation and prothrombin G2021A are among the most requested genetic tests in clinical molecular diagnostics laboratories. Their medical utility was recently reviewed (17). In isolation, the presence of these single point mutations is not diagnostic for disease nor do they necessarily predict susceptibility or future likelihood of disease. They are only useful when considered in the context of personal and family history, coexisting conditions, physical symptoms, and other laboratory tests. In some cases, the presence of these mutations with demonstrated thrombotic disease will suggest more aggressive therapy. Of particular interest is a potential for using these tests as an indicator of risk for women receiving oral contraceptive or hormone replacement therapy. At present, screening of healthy women is not recommended since it would deny oral contraceptives to about $5 \%$ to $10 \%$ of Caucasian women who would then use less effective means of preventing pregnancy. It has been calculated that there would be more deaths from complications of unwanted pregnancies than would be prevented by avoiding thrombotic complications related to factor $\mathrm{V}$ Leiden.

New methodologies have made these assays reliable, convenient, and cost-effective. As an example, Figures 1 and 2 show the performance of the READIT system for genotyping samples for the factor V Leiden and prothrombin point mutations. Separation is sufficient to clearly distinguish the genotypic populations by greater than six standard deviations around the population means. This provides an unprecedented level of accuracy and confidence in the genotype calls.

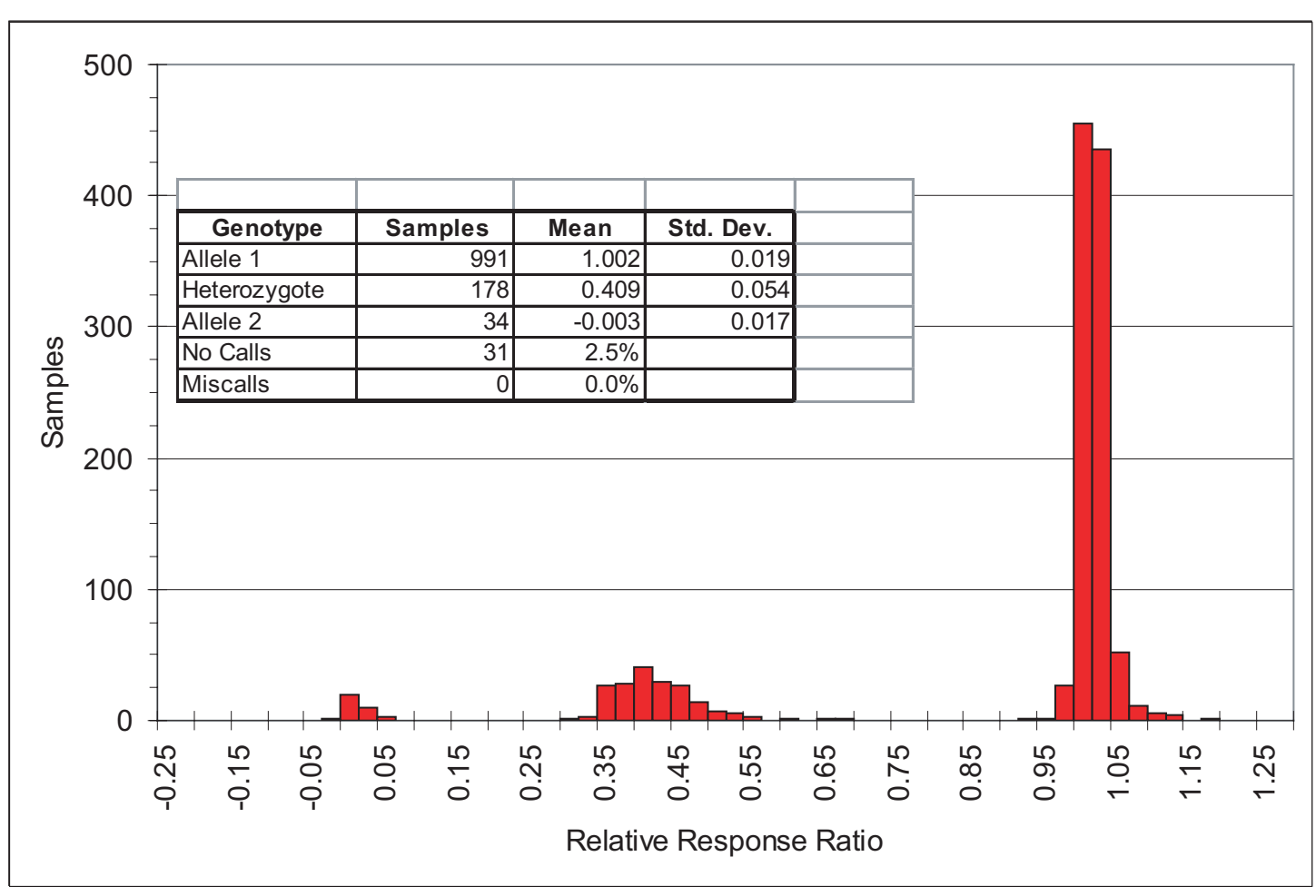

Figure 1. SNP genotyping of 1234 discrete samples for the factor V Leiden G1691A polymorphism. Sample populations clustered around Relative Response Ratios (signal output) of 1.0, 0.4 , and 0.0 are homozygous wild-type, heterozygote, and homozygous mutant, respectively. 
Table 1. Partial Test Menu from a Major Reference Laboratory

\begin{tabular}{|llc|}
\hline Assay & \multicolumn{1}{c}{ Clinical Utility } & Volume* $^{*}$ \\
\hline Cystic Fibrosis & Diagnostic, prenatal, and carrier screening; reproductive counseling & 13000 \\
CYP2D6 & Reduce risk of adverse drug reactions based on genotype & 150 \\
Factor V Leiden & Prevention of thrombotic events in at-risk individuals & 6000 \\
Factor II (Prothrombin) & Prevention of thrombotic events in at-risk individuals & 2000 \\
Fragile X Syndrome & Identify cause of mental retardation; reproductive counseling & 900 \\
Hereditary Hemochromatosis & Prevent organ damage from iron overload & 1500 \\
Jewish Genetic Diseases & Carrier screening and reproductive counseling & 400 per assay \\
Factor V HR2 Allele & Prevention of thrombotic events in at-risk individuals & 500 \\
Non-classical Congenital & Symptoms can be mitigated by early treatment & 400 \\
Adrenal Hyperplasia (CAH) & & 100 \\
Medium-chain Acyl CoA & Reduce morbidity and mortality through dietary measures in homozygotes \\
Dehydrogenase & & \\
Deficiency (MCAD) & & \\
*Approximate or estimated volumes per month for assays currently performed or under development & \\
\hline
\end{tabular}

\section{Testing for Predisposition to Cancer}

One of the promises of genetic testing is to help identify individuals at high risk of developing serious and incurable disease to allow for prophylactic treatment. This is perhaps the most controversial adult application of molecular diagnostics. Typi- cally, the disease is cancer, and prevention requires more aggressive monitoring or prophylactic removal of the target organ(s). On the one hand, the patient is faced with possible death from an incurable disease; on the other, disfigurement and/or severe functional impairment resulting from preventive surgery. Clinical decision-making is especially difficult since independent ver-

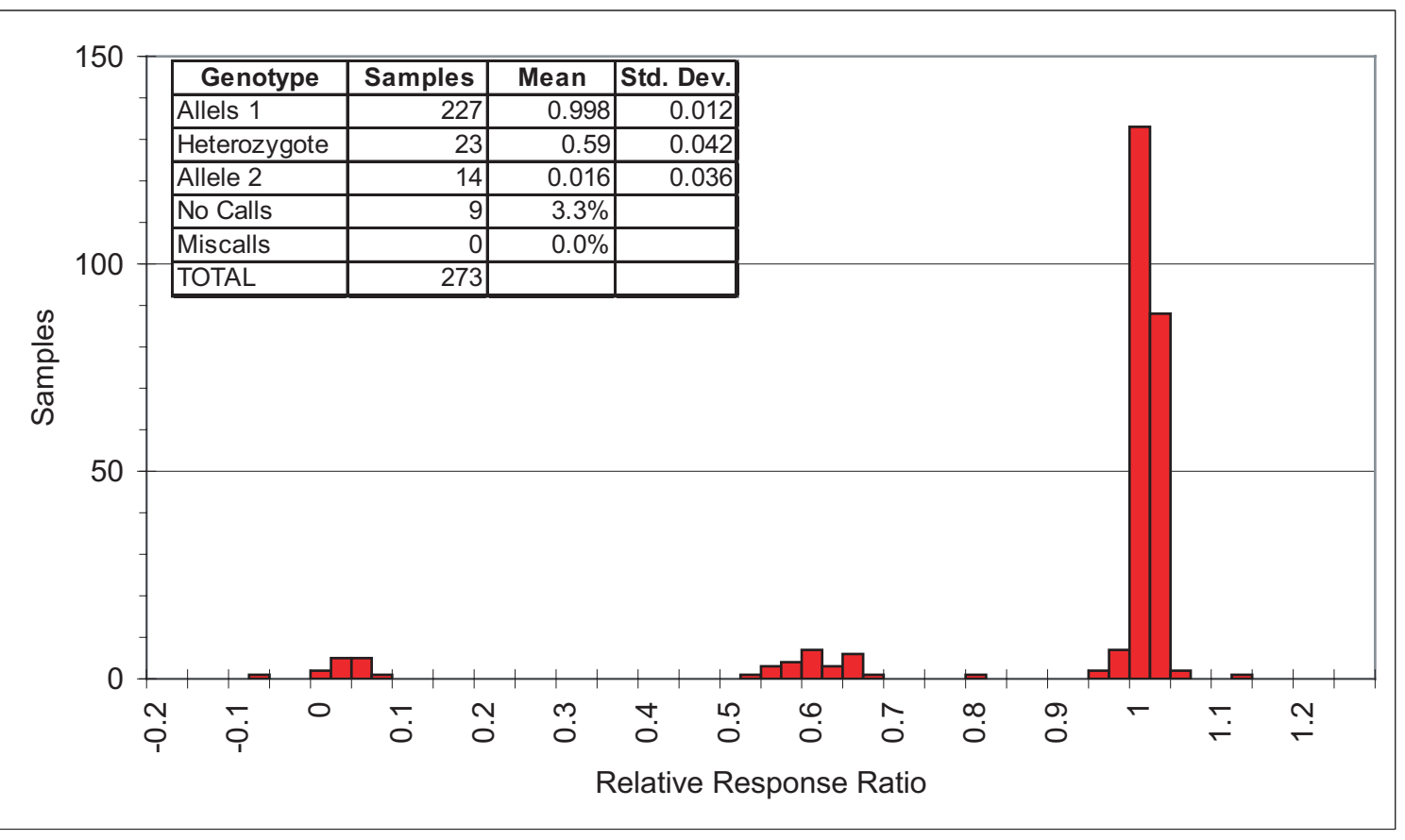

Figure 2. SNP genotyping of 272 discrete samples for the prothrombin or factor II G2021A polymorphism. Sample populations clustered around Relative Response Ratios (signal output) of 1.0, 0.6 , and 0.0 are homozygous wild-type, heterozygote, and homozygous mutant, respectively. 
ification of the test result is impossible to obtain as the disease is not actually present at the time of testing and no independent tests to assess risk are available.

Two examples of predisposition testing have been in use for some time, the adenomatous polyposis coli $(A P C)$ gene associated with colorectal cancer and the BRCA1 and BRCA2 genes associated with breast and ovarian cancers. While not strictly SNP assays, they do illustrate the challenges inherent in this approach to molecular diagnostic testing.

Familial adenomatous polyposis is an autosomal dominant disease caused by a germline mutation in the $A P C$ gene (5). It will typically lead to colorectal cancer by the sixth decade of life in all affected individuals unless prophylactic colectomy is performed. Genetic testing has been available since 1994. The test can identify $A P C$ mutations in about $80 \%$ of affected families, and there is almost $100 \%$ accuracy in differentiating affected from unaffected family members in kindreds with a characterized mutation. From a genetic and analytical standpoint, this would seem to be close to an ideal test. However, the researchers at Johns Hopkins University (Baltimore, MD, USA) and LabCorp (Research Triangle Park, NC, USA) found that other factors compromised the efficacy of this testing. Only $17 \%$ of the patients provided written informed consent and only $19 \%$ received genetic counseling prior to testing. In $32 \%$ of the cases, clinicians misinterpreted the test results. The most common error was that physicians didn't understand that the testing did not identify all clinically important mutations and that a patient who tested negative could still harbor a mutation of the $A P C$ gene that would ultimately lead to the development of colon cancer. This study shows that effective use of genetic tests to determine predisposition to disease will require that we train medical doctors and laboratorians in the complexities of genetic testing and ensure that every patient receives counseling as to the implications of this testing and gives informed consent. This issue has captured the attention of the molecular pathology community and the regulatory agencies that govern laboratories offering these testing services.

$B R C A 1$ and BRCA2 testing for cancer susceptibility raises more complex issues than $A P C$ gene testing. Myriad Genetics (Salt Lake City, UT, USA), the company that has pioneered discovery and offers testing for $B R C A 1$ and $B R C A 2$ mutations, estimates that mutations in these genes are responsible for $7 \%-10 \%$ of all breast and ovarian cancers and that women who express deleterious mutations have a lifetime risk of breast cancer between $56 \%$ and $87 \%$ and a lifetime risk of ovarian cancer between $27 \%$ and $44 \%$. There is also evidence linking these genes to risk for colorectal cancer. Deleterious mutations are more prevalent in some populations, especially those of Ashkenazi ancestry. In addition, not all sequence deviations from the wildtype genes have been characterized. Like the $A P C$ gene, kindreds will have the same mutation, and differential testing is very effective in distinguishing affected from unaffected family members. In the case of $B R C A$ testing, however, the prognosis is more complex and the patient has multiple options, ranging from watchful waiting to prophylactic oophorectomy and mastectomy, to consider. The need to help patients make informed choices regarding testing, interpretation, and follow-up is even more critical. Fortunately, objective published guidance from the Cancer Studies Consortium is available (2).
Table 2. Predictive Value of Testing for a Hypothetical Haplotype

\begin{tabular}{|c|c|c|c|c|}
\hline & \multicolumn{2}{|c|}{$\begin{array}{c}\text { Phenotype } \\
\text { (Susceptibility to Disease) }\end{array}$} & \multirow[b]{2}{*}{ Total } \\
\hline & & + & - & \\
\hline \multirow[t]{3}{*}{ Haplotype } & + & 160 & 9980 & 10140 \\
\hline & - & 40 & 89820 & 89860 \\
\hline & Total & 200 & 99800 & 100000 \\
\hline
\end{tabular}

\section{Using Haplotypes to Determine Susceptibility to Multifactorial Disease}

To many, this category represents the greatest potential for long-term value from SNP genotyping. The previous examples rely on association, or identification of a polymorphism directly associated with a functional gene. Such associations allow a SNP to be directly associated with gene function and ultimately to phenotype. As we have seen, the complex overlapping pathways and environmental interactions that constitute living organisms make phenotypic predictions based on even functional mutations problematic. The proposed challenge is to identify haplotypes, or combinations of SNPs, that determine susceptibility to disease phenotypes. Massive studies are required to search for haplotypes that are in linkage disequilibrium or appear more frequently than expected in a target population.

In the absence of a functional link between the haplotype and disease, researchers must rely on statistical methods to make a connection. Unfortunately, establishing a firm statistical relationship does not mean the presence of the haplotype will be a useful diagnostic tool. These issues were recently reviewed in the context of medical care and are illustrated by the following hypothetical example (6).

Our hypothetical haplotype is statistically linked with a particular disease in a target population. The prevalence (proportion of people in the population who have the disease) is 2 per 1000 or $0.2 \%$. The haplotype is found in $80 \%$ of those who will ultimately develop the disease (sensitivity) and in only $10 \%$ of unaffected individuals (specificity). Assuming the assay for the haplotype is perfect and error-free, the testing results on 100000 random individuals can be predicted as shown in Table 2. Of the 10140 individuals who express the haplotype, only 160 or $1.6 \%$ would actually develop the disease. Detection of this haplotype would have no value as a predictive diagnostic test.

In reality, the statistical association with disease is not likely to be as strong as that described in our hypothetical example. For example, a Finnish group found a haplotype comprising two polymorphisms to be statistically related $(P<0.005)$ to acute myocardial infarction (AMI) and coronary thrombosis (CT), especially in men younger than 55 years old (12). In this population the sensitivity or proportion of affected men expressing the haplotype was $59 \%$ for AMI and $70 \%$ for CT. The specificity, or proportion of the control group (men who died of non-cardiac causes) who expressed the haplotype, was 20\%. While statistically valid and scientifically significant, it is unlikely that testing for this haplotype will prove to be of diagnostic value.

The extreme difficulty of identifying and determining utility of haplotypes in complex disease is exemplified by the work of 
Graeme Bell's lab with type 2 (non-insulin dependent) diabetes mellitus (7). This disease affects approximately $4 \%$ of the world's population and is believed to be associated with a combination of both genetic and environmental factors. This paper is instructive, if for no other reason than because it illustrates the tremendous effort required to do this type of work. The target population was a group of Mexican Americans; however, other data also reflect various European, Asian, and Native American populations. Ultimately, a haplotype consisting of three polymorphisms was identified in the gene for calpain-10, a member of the calpain-like cyteine protease family not previously associated with this disorder, that linked to type 2 diabetes in the target population. At present, there is no demonstrated functional association between this gene and type 2 diabetes. The authors estimate the population-attributable risk or the proportion of the study population that would not be affected if the risk factor were not present: 14\% for the Mexican Americans and 4\% for Europeans. These findings are of tremendous scientific value. Diagnostically, the risk associated with this haplotype is strongly population dependent and well below the level of diagnostic utility even for the Mexican American population. Perhaps in the future it will provide a useful clue in determining the mechanism for the disease or a means of stratifying patients into therapeutic categories.

\section{CONCLUSIONS}

We have made incredible progress in sequencing the human genome in a relatively short period of time. The discovery, mapping, and cataloging of SNPs has accompanied this effort and provides an important tool in our efforts to use genomic sequence as a tool in the ongoing effort to understand and diagnose disease. For the first time, we have the potential for genetically identifying individuals at risk for developing a disease before it occurs. This capability is especially significant for diseases such as certain cancers that are not readily treatable, but where prophylactic measures are available. However, the association between these genetic markers and the presence or susceptibility to disease is often not completely defined, even when the genotype has been demonstrated to be a causative factor in the disorder. This uncertainty complicates the use of genetic testing in clinical molecular diagnostics. The complexity increases substantially when haplotypes are used to predict susceptibility where there is no functional association between the genotype and disease phenotype. At present, even the best candidate haplotype assays fall short of the predictive value required for practical use in a clinical environment.

This is not intended to be a pessimistic view of the longer term potential for SNP assays becoming useful tools in diagnostic medicine. Not included in this discussion is the potential of pharmacogenomics, or the use of SNP assays to predict the most effective member of a therapeutic family for a particular individual. This testing, also called personalized medicine, may become the dominant clinical application for genotyping over the next five to ten years. Over time, we will develop the more detailed cataloguing of useful polymorphisms in the human genome that will provide the sensitivity and specificity required for useful diagnostic testing.

\section{REFERENCES}

1.Buetow, K.H., M. Edmonson, R. MacDonald, R. Clifford, P. Yip, J. Kelley, D.P. Little, R. Strausberg, et al. 2001. High-throughput development and characterization of a genomewide collection of gene-based single nucleotide polymorphism markers by chip-based matrix-assisted laser desorption/ionization time-of-flight mass spectrometry. Proc. Natl. Acad. Sci. USA 98:581-584

2.Burke, W., M. Daly, J. Garber, J. Botkin, M.J.E. Kahn, P. Lynch, A. McTiernan, K. Offit, et al. 1997. Recommendations for follow-up care of individuals with an inherited predisposition to cancer II BRACA1 and BRCA2. JAMA 277:997-1003.

3.Daly, M.J., J.D. Rioux, S.F. Schaffner, T.J. Hudson, and E.S. Lander. 2001. High-resolution haplotype structure in the human genome. Nat. Genet. 29:229-232.

4.De Stefano, V. I. Martinelli, P. Mannucci, K.L. Paciaroni, P. Chiusolo, I. Casorelli, E. Rossi, and G. Leone. 1999. The risk of recurrent deep venous thrombosis among heterozygous carriers of both factor V Leiden and the G2021A prothrombin mutation. N. Engl. J. Med. 341:801-806.

5.Giaardiello, F.M., J.D. Brensinger, G.M. Petersen, M.C. Luce, L.M. Hylind, J.A. Bacon, S.V. Booker, R.D. Parker, et al. 1997. The use and interpretation of commercial $A P C$ gene testing for familial adenomatous polyposis. N. Engl. J. Med. 336:823-827.

6.Hoffrage, U., S. Lindsey, R. Hertwig, and G. Gigerenzer. 2000. Communicating statistical information. Science 290:2261-2262.

7.Horikawa, Y., N. Oda, N.J. Cox, L. Xiangquan, M. Orho-Melander, M. Hara, Y. Hinokio, T.H. Lindner, et al. 2000. Genetic variation in the gene encoding calpain-10 is associated with type 2 diabetes mellitus. Nat. Genet. 26:163-175.

8.http://snp.cshl.org/.

9.http://www.ncbi.nlm.nih.gov/.

10.Johnson, G.C.L., L. Esposito, B.J. Barratt, A.N. Smith, J. Heward, G. Di Genova, H. Ueda, H.J. Cordell, et al. 2001. Haplotype tagging for the identification of common disease genes. Nat. Genet. 29:233-237.

11.Matyas, G., C. Giunta, B. Steinmann, J.P. Hossle, and R. Hellwig. 2002. Quantification of single nucleotide polymorphisms: a novel method that combines primer extension assay and capillary electrophoresis. Hum. Mutat. 19:58-68.

12.Mikkelsson, J., M. Perola, A. Penttilä, and P.J. Karhunen. 2001. Platelet Glycoprotein Ib? HPA-2 Met/VNTR B haplotype as a genetic predictor of myocardial infarction and sudden cardiac death. Circulation 104:876-880.

13.Olivier, M., A. Aggarwal, J. Allen, A.A. Almendras, E.S. Bajorek, E.M. Beasley, S.D. Brady, J.M. Bushard, et al. 2001. A high-resolution radiation hybrid map of the human genome draft sequence. Science 291:1298-1302.

14.Patil, N., A.J. Berno, D.A. Hinds, W.A. Barrett, J.M. Doshi, C.R. Hacker, C.R. Kautzer, D.H. Lee, et al. 2001. Blocks of limited haplotype diversity revealed by high-resolution scanning of human chromosome 21. Science 294:1719-1723.

15.Reich, D.E., M. Cargill, S. Bolk, J. Ireland, P.C. Sabeti, D.J. Richter, T. Lavery, R. Kouyoumjian, et al. 2001. Linkage disequilibrium in the human genome. Nature 411:199-204

16.Rioux, J.D., M.J. Daly, M.S. Silverberg, K. Lindblad, H. Steinhart, Z. Cohen, T. Delmonte, K. Kocher, et al. 2001. Genetic variation in the $5 \mathrm{q} 31 \mathrm{cy}-$ tokine gene cluster confers susceptibility to Crohn disease. Nat. Genet. 29:223-228.

17.Selgsonh, U. and A. Lubetsky. 2001. Genetic susceptibility to venous thrombosis. N. Engl. J. Med. 344:1222-1231.

18.Syvanen, A-C. 2001. Accessing genetic variation: genotyping single nucleotide polymorphisms. Nat. Rev. Genet. 2:930-942.

19.Voelkerding, K. 1996. Resistance to activated protein C and a novel factor V gene mutation. Clin. Lab. Med. 16:169-186.

\section{Address correspondence to:}

Dr. Richard S. Schifreen

Promega Corporation

2800 Woods Hollow Road

Madison, WI, 53711

e-mail: rschifre@promega.com 